\title{
Effect of Reaction Time on the Morphology of Tungsten Disulfide Nanosheets by Solvothermal Method
}

\author{
Deqing ZHANG, Tingting LIU \\ School of Materials Science and Engineering \\ Qiqihar University \\ Qiqihar, China \\ e-mail: zhdqing@163.com, 976115939@qq.com
}

\begin{abstract}
In this work, a modified solvothermal method has been developed to synthesize few layers of tungsten disulfidenanosheets(WS2-NS). In order to determine the effect of reaction time on the morphology of the product in the preparation of ultrathin tungsten disulfide nanosheets, we prepared samples at different reaction times. As-prepared WS2 samples were characterized by XRD, TEM, and Raman spectrum. The results showed that the obtained $\mathrm{WS}_{2}-\mathrm{NS}$ were consisted of 1-10 atomic layers. Importantly, the effects of reaction time on the morphology formation of $\mathrm{WS}_{2}-\mathrm{NS}$ were investigated.
\end{abstract}

Keywords-solvothermal synthesis; $\quad W S_{2}$-Nanosheets;
rphological differences

\section{INTRODUCTION}

In recent years, fullerene-like transition metal disulfides (TMDS) has attracted widely attention [1]. As a representative substance of TMDS, $\mathrm{WS}_{2}$ can be chemically or physically processed into two-dimensional nanosheet structure [2]. The tungsten disulfide nanosheet having an atomic thickness exhibits a unique property [3] different from that of the bulk materials [4]. After the bulk material has been peeled off into a single layer or several layers of ultrathin two-dimensional sheet material [5]. these materials retain most of the properties of the bulk material. at the same time, due to the quantum effect, these ultra-thin twodimensional material showed new performance [6]. Due to the peculiar electron configuration and high specific surface area, $\mathrm{WS}_{2}-\mathrm{NS}$ has potential application in catalytic hydrogen production [7], electrode devices [8] and energy storage devices [9].

To date, various methods have been reported to prepare the $\mathrm{WS}_{2}-\mathrm{NS}$, including mechanical exfoliation, chemical exfoliation, chemical vapor deposition and hydrothermal method. Late et al [10] obtained the few layers $\mathrm{WS}_{2}-\mathrm{NS}$ by repeatedly folding a piece of adhesive scotch tape with $\mathrm{WS}_{2}$ powder. Coleman et al [11] developed a reliable surfactantassisted liquid exfoliation method to produce $\mathrm{WS}_{2}-\mathrm{NS}$. In the present work, in order to meet the requirements of $\mathrm{WS}_{2^{-}}$ NS used in potential fields, a one-step solvothermal method was proposed to stably and efficiently prepare $\mathrm{WS}_{2}-\mathrm{NS}$. The effects of reaction time on the morphology and structure were studied, and the possible growth mechanism was discussed.

\section{EXPERIMENTAL}

\section{A. Synthesis of $W_{2}$ nanosheets}

All chemicals were analytical degree and used without further purification. In a typical process, $0.169 \mathrm{~g}$ of tungsten chloride $\left(\mathrm{WCl}_{6}\right)$ and $0.576 \mathrm{~g}$ of sodium diethyldithiocarbamate and $0.09 \mathrm{~g}$ of sodium cetvltrimethylammonium bromide were dissolved in $36 \mathrm{ml}$ of absolute ethanol, and the resulting solution was stirred for $30 \mathrm{~min}$ and transferred to a $100 \mathrm{ml}$ of para-polyphenylene (PPL) lined stainless steel autoclave which was afterwards sealed tightly, and heated at $220^{\circ} \mathrm{C}$ for 8 hours, 0 hours, 12 hours, 18 hours. Then the reaction kettle was taken out and cooled to room temperature. The black precipitates were retrieved from the solution by centrifugation and washed with distilled water and ethanol for several times, and finally dried in vacuum at $50{ }^{\circ} \mathrm{C}$ for $6 \mathrm{~h}$. Since the sample prepared at 8 hours was still granular, the starting material did not react, so we will not discuss it in the following. To better distinguish the samples, samples prepared by heating for 10 hours , 12 hours and 18 hours are labeled as Sample I , sample II, and sample III in turn. In our experiment, the reaction routes for the synthesis of $\mathrm{WS}_{2}$ could be expressed as follows [12]:

$$
\begin{gathered}
\mathrm{W}^{6+}+\mathrm{R}=\mathrm{W}^{4+}+\mathrm{R}^{*} \\
\mathrm{~W}^{4+}+\mathrm{S}=\mathrm{WS}_{2}+\mathrm{S}^{*} \\
\mathrm{~W}^{6+}+\mathrm{RS}=\mathrm{WS}_{2}+\mathrm{RS}^{*}
\end{gathered}
$$

Where $\mathrm{R}$ is a reducing agent, $\mathrm{S}$ is a vulcanizing agent, $\mathrm{RS}$ also acts as a vulcanizing agent having a reducing property, $\mathrm{R}^{*}, \mathrm{~S}^{*}$ and $\mathrm{RS}^{*}$ are generated are substances after the reaction.

\section{B. Charaterization}

The X-ray diffraction(XRD) patterns were recorded using German Bruker-AXS D8 X-ray diffractometer with $\mathrm{Cu} \mathrm{K} \alpha$ radiation $(\lambda=0.1546 \mathrm{~nm})$. The morphology of the as- 
synthesized products were characterized by transmission electron microscopy (TEM, Hitachi, H-7650).Raman spectroscopy measurements were carried on a Lab RAM HA Evolution.

\section{RESULTS AND DISCUSSION}

\section{A. Morphology ang Structure of $W S_{2}$ nanosheets}

The XRD patterns of $\mathrm{WS}_{2}$-nanosheets were showed in Figure 1. In order to study the effect of reaction time on the composition of the samples. As presented in Figure 1, A, B, and $\mathrm{C}$ are the XRD patterns of the $\mathrm{WS}_{2}$ heterostructures prepared heated at $220^{\circ} \mathrm{C}$ for 18 hours, 12 hours, 10 hours respectively. When the diffraction angle is located in $15^{\circ}, 28^{\circ}$, or $39^{\circ}$, there aren't obvious diffraction peaks. The curve of $\mathrm{C}$ is the smoothest and the degree of crystallization is also the best. All samples present representative peaks indexed to the hexagonal $\mathrm{WS}_{2}$ structure (JCPDS card No.87-2417). It shows the similar XRD patterns whether the reaction time is 10 hours, 12 hours, or 18 hours indicating that the reaction time has no effect on crystallization of $\mathrm{WS}_{2}-\mathrm{NS}$.

The morphology of $\mathrm{WS}_{2}-\mathrm{NS}$ was characterized by TEM. In Fig.2, (A) is the TEM image of sample I with the reaction time of $10 \mathrm{~h}$; Fig (B) is the TEM image of the product obtained with the reaction time of $12 \mathrm{~h}$; Fig (C) and (D) for the reaction time is $18 \mathrm{~h}$ TEM image obtained. Transmission electron microscopy images of the three samples at $200 \mathrm{~nm}$ were compared. The comparison shows that the reaction time in the hydrothermal preparation of $\mathrm{WS}_{2}$ has a certain

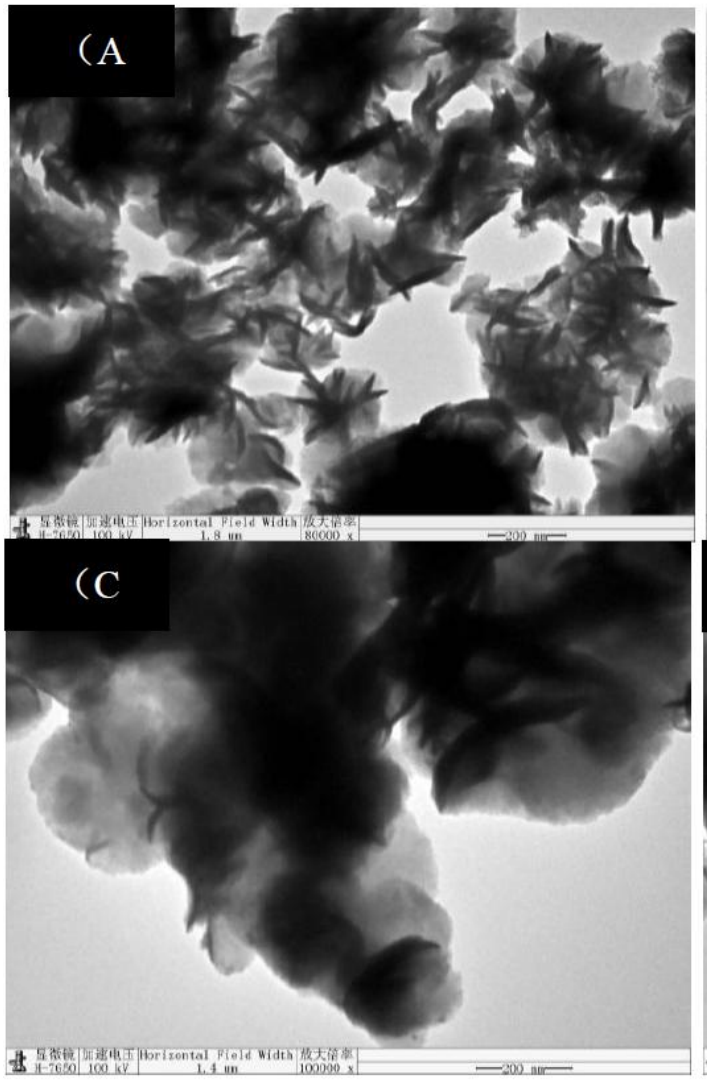

influence on the morphology. From the transmission electron microscope image it can be seen that after the reaction time is increased, the grain grow and the agglomeration became obvious. The transparency and the integrity of the nanosheets were getting worse. Arrangement becomes loose, the phenomenon of incomplete reaction also becomes obvious. And the reaction time is 8 hours the raw material did not react, no nanosheet generation. Thus proving that in other reaction conditions are the same, in the case of reaction time is $10 \mathrm{~h}$, the morphology of the ultrathin tungsten disulfide nanosheet is the best.

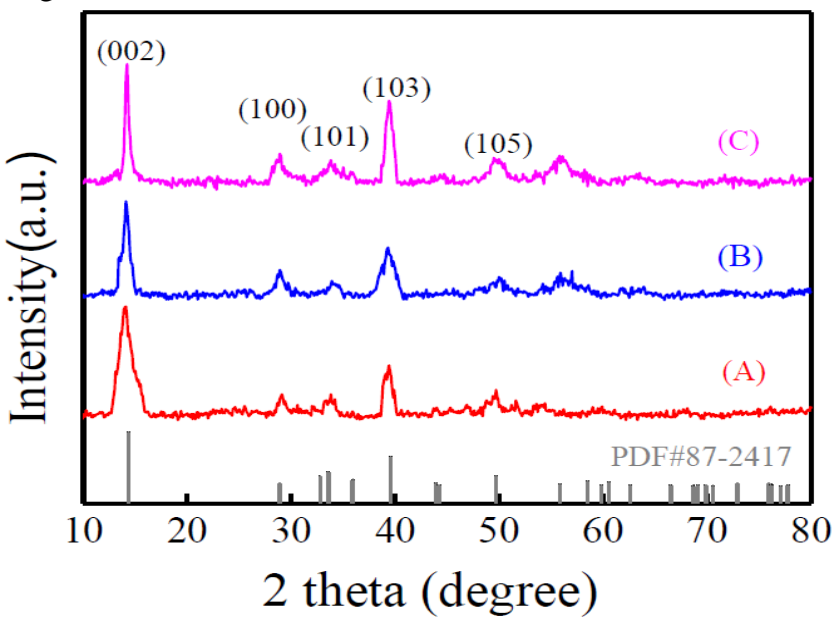

Figure 1. XRD patterns of (A) sample III , (B) sample II and (C) sample I.

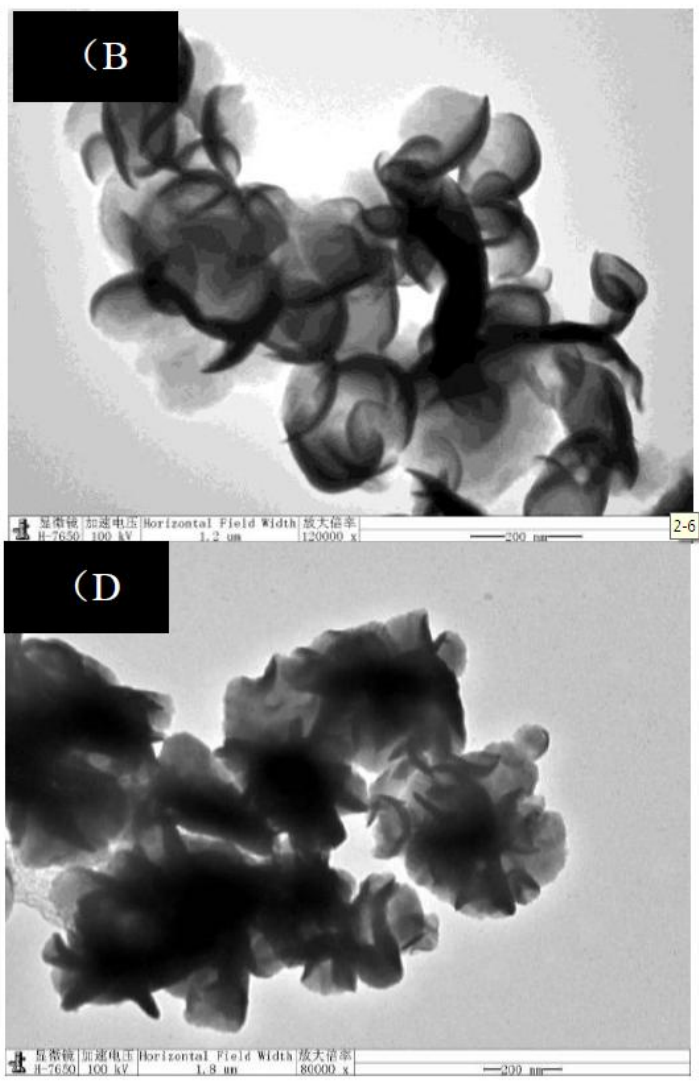

Figure 2. TEM images of (A) sample I, (B and C) sample II and (D) sample III. 
Raman spectroscopy was further applied to characterize the layered structure of the $\mathrm{WS}_{2}$. According to previous reports, the bulk crystal $\mathrm{WS}_{2}$ shows two Raman peaks at 355 and $421 \mathrm{~cm}^{-1}$ corresponding to the well know active Raman modes in-plane $E_{2 g}^{1}$ and out-of-plane $A_{1 g}$. With the decrease of the number of crystals layers, the two peaks are red-shifted and blue-shifted respectively and close to each other. As show in Figure 3, the two peaks of sample III appeared at 355.1 and $420.2 \mathrm{~cm}^{-1}$, which is consistent with bulk crystal $\mathrm{WS}_{2}$. The sample II is a plate-like structure composed of regularly arranged nanosheets and possesses certain thickness as compared with few layer nanosheets, so these two peaks appeared at 359.1 and $420.2 \mathrm{~cm}^{-1}$ respectively. The $E_{2 g}^{1}$ peak and $A_{1 g}$ peak of sample I appeared at 362.1 and $417.2 \mathrm{~cm}^{-1}$ respectively and Raman shift between these two peaks is $55.1 \mathrm{~cm}^{-1}$. Referring to Late et al, the thickness of sample I is about 1-10 atomic layers. Hence, independent of the TEM measurements, these results provide an additional confirmation of the few layer characteristics of sample I .

\section{B. Experimental Mechanism}

With tungsten chloride as the tungsten source and sodium diethyldithiocarbamate as the sulfur source of the reaction, cetyl trimethyl ammonium bromide as a surfactant, the crystal growth mechanism in the solution is often very complicated, and the actual solution crystallization process is still unclear [13]. In our experiments, the mechanism for the synthesis of tungsten disulfide $\left(\mathrm{WS}_{2}\right)$ crystals is the oxidation-reduction reaction. $\mathrm{WS}_{4}{ }^{2-}$ as an intermediate product in the synthesis process formed by the rapid reaction of tungsten hexachloride $\left(\mathrm{WCl}_{6}\right)$ and sodium diethyldithiocarbamate in anhydrous ethanol solution. At the initial stage of synthesis, a large amount of tiny crystal core nanoparticles of tungsten disulfide $\left(\mathrm{WS}_{2}\right)$ first formed. When the free enthalpy of this system became negative, the gain of the surface energy compensates for the energy of the lattice growth [14], the surfactant then acts, CTAB will selfassemble to form micelles. As presented, nano-CTAB molecules tend to form lamellar micelles. In order to maintain the energy of the low system [15], the layered micelles are aggregated together to form ordered monodisperse nanocrystals, spontaneously with the presence of van der Waals forces between the surfactants of CTAB, leading to the assembly of the ordered structure [16]. The ordered assembly provides a specific microenvironment for chemical reactions, where the resulting $\mathrm{WS}_{2}$ petal-shaped lamellar nanocrystals gradually grew and stacked together to eliminate surface energy, as shown in Figure 4.

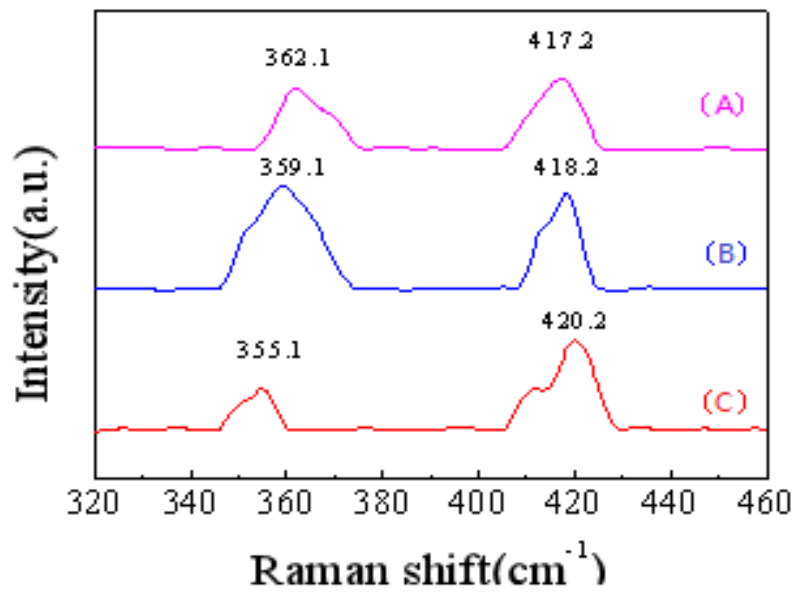

Figure 3. Raman spectra of (A) sample I, (B) sample II and (C) sample III.

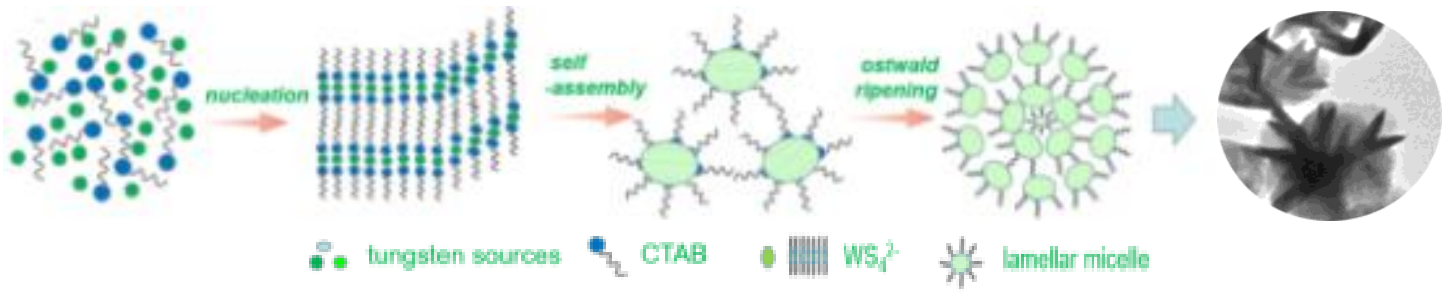

Figure 4. Illustration of WS2 reaction mechanism diagram.

\section{CONCLUSIONS}

In summary, $\mathrm{WS}_{2}$-NS were successfully prepared by a simple one-step solvothermal process. The experimental results of the samples prepared at different reaction times show that the reaction time plays an important role in the growth of $\mathrm{WS}_{2}-\mathrm{NS}$. When reaction time was $10 \mathrm{~h}, \mathrm{WS}_{2}$ presented a sheet-like structure. Furthermore, a possible growth mechanism was proposed to explain the formation of $\mathrm{WS}_{2}-\mathrm{NS}$. Considering the simple synthetic process and thinness of $\mathrm{WS}_{2} \mathrm{NS}$, we believe this work will open an avenue for industrial production of $\mathrm{WS}_{2}-\mathrm{NS}$.

\section{ACKNOWLEDGMENT}

This work was supported by the National Natural Science Foundation of China under Grant No.51272110, 51772160.

\section{REFERENCES}

[1] Seo. J.W, Jun, Park. S.W., Nah. H, Moon. T, et al, Two-dimensional nanosheet crystals. Angew Chem Int Ed. 46(46), 8828-31(2007).

[2] Novoselov. K.S, Jiang D, Schedin. F, Booth. T.J, Khotkevich. V.V, et al, Two-dimensional atomic crystals. Proc Natl Acad Sci U SA. 102(30), 10451-3(2005).

[3] Lee. C, Li. Q, Kalb. W, Liu. X.Z, Berger. H, et al, Frictional characteristics of atomically thin sheets. Sci. 328(5974), 76-80(2010). 
[4] Huang. X, Yin. Z, Wu. S, Qi. X, He. Q, Zhang. Q, et al, Graphenebased materials: synthesis, characterization, properties, and applications. Small. 7(14), 1876-1902(2011).

[5] Smith. R.J, King. P.J, Lotya. M, Wirtz. C, Khan. U, De. S, et al, Large-scale exfoliation of inorganic layered compounds in aqueous surfactant solutions. Adv Mater. 23(34), 3944-8(2011).

[6] Gordon. R.A, Yang. D, Crozier. E.D, Jiang. D.T, Frindt. R.F, Structures of exfoliated single layers of $\mathrm{WS}_{2}, \mathrm{MoS}_{2}$, and $\mathrm{MoSe}_{2}$, in aqueous suspension. Phys rev b. 65(12), 125407(2002).

[7] Novoselov. K.S Geim. A.K, Morozov. S.V, Jiang D, Zhang. Y, et al. Electric field effect in atomically thin carbon films. Sci. 306(5696), 666-9(2014)

[8] Niederberger M, CÖlfen H. Phys Chem Chem Phys. 8:3271-87(2006).

[9] Zeng. Z, Yin. Z, Huang. X, Li. H, He. Q, Lu. G, et al, Single-layer semiconducting nanosheets: high-yield preparation and device fabrication.. Angew Chem. 50(47), 11093-7(2011).
[10] Late D J, Temperature dependent phonon shifts in single-layer $\mathrm{WS}_{2}$. ACS Appl Mater \& Interfaces. 6(2): 1158-1163(2014).

[11] Pacholski C, Kornowski A, Weller H. Angew Chem Int Ed.. 41:11889(2002).

[12] TIAN Ye, HE Tian, SHANG Jing, et al. Synthesis of $\mathrm{MoS}_{2}$ layered material by hydrothermal method and its structural characterization [J]. Acta Chim Sinica. 62 (18): 1807-1810(2004).

[13] CAO Si, Liu Tang, Hussain S, et al. Hydrothermal synthesis of variety low dimensional $\mathrm{WS}_{2}$, nanostructures[J]. Mater Letters. 129(1):205-208(2014).

[14] Miremadi. B.K, Morrison. S.R, The intercalation and exfoliation of tungsten disulfide. J Appl Phys. 63(10), 4970-4974(1988).

[15] Bhandavat. R, David. L, Singh. G, Synthesis of surfacefunctionalized $\mathrm{WS}_{2}$ nanosheets and performance as li-ion battery anodes. J Phys Chem Letters. 3(11), 1523-1530(2012).

[16] Zeng. Z, Huang. X, Li YD. Chem Eur J. 12:6552-8(2006). 